\title{
SUR QUATRE
}

\section{LIMONIA (DICRANOMYIA) EUROPÉENNES PEU CONNUES}

[Diptera, Tipulidae]

par A. G. B. Thomas ${ }^{1}$.

La plupart des espèces européennes de Limonia (Dicranomyia) sont assez aisément identifiables, à l'état imaginal, grâce essentiellement à la révision des Limoniini par Lackschewitz et Pagast [1940 à 1942]. Toutefois, les genitalia de quelques-unes des espèces redécrites ne sont pas dessinés. Les descriptions antérieures de quatre de ces espèces sont soit dépourvues de figures, soit accompagnées de schémas sommaires et depuis n'ont pas fait l'objet de mise au point.

Une étude sur les Diptères Tipulidae des Pyrénées m'a amené à déterminer, avec quelques difficultés, ces quatre espèces et il m'a paru utile d'en représenter en détail les genitalia.

Je remercie M. A. M. Hutson (British Museum, Londres) pour son aimable collaboration.

\section{L. (D.) stigmatica Meigen 1830 .}

Figures partielles des genitalia : Edwards [1938] - repris par Coe [1950] - et Bangerter [1948]. Les figures de De Meijere [1919] sont plus complètes mais réalisées avec les moyens optiques de l'époque. Le schéma de Pierre [1924] est à peu près inutilisable.

Après examen des figures 1 à 6 , M. Hutson a conclu à l'identité de l'espèce pyrénéenne et des spécimens de la collection Edwards. En particulier, le court appendice plumeux situé entre les deux épines dorsales de la pince génitale (fig. 2, 3, 4 et 6) - absent sur la figure $5 \mathrm{f}$ d'Edwards - existe sur l'un des specimens récoltés

1. Laboratoire d'Hydrobiologie, Université Paul-Sabatier, 118, route de Narbonne, 31-Toulouse. 
par cet auteur. Cet appendice peu chitinisé, très fragile, est dirigé vers l'intérieur et doit se briser fréquemment, en particulier lors de l'accouplement (il n'est pas représenté non plus par De MeiJere ni par Bangerter). Sur le spécimen figuré par Edwards, il en subsiste la base (A. M. Hurson in litt.).

Provenance : ruisseau des Pins à $1900 \mathrm{~m}$ dans le bassin de la Neste d'Aure (Hautes-Pyrénées), 18-IX-1967 (1 $\delta, 1$ \&) et 26-X-1967 (1 \&).

Cette espèce est nouvelle pour la faune de France.

\section{L. (D.) lackschewitzi Edwards 1928.}

La description d'Eowards n'est pas accompagnée de figures des genitalia. L'auteur a seulement indiqué que celles-ci devaient être exécutées par Lackschewitz dans sa révision des Limoniinae paléarctiques, ce qui n'a pas été réalisé par ce dernier, ni effectué depuis.

L'exemplaire dessiné ici (fig. 7 à 11) appartient à la collection Edwards du British Museum; c'est le seul $\hat{o}$, en dehors du type (A. M. Hutson in litt.).

Provenance : Corte-R-Restonica (Corse), 10-25-IV-1928 (B M 1928-214).

\section{L. (D.) halterella Edwards 1921.}

Figures partielles d'Edwards [1938], reprises par Coe [1950]. Le schéma de Pierre [1924] peut rappeler plusieurs espèces de Dicranomyia - mais ne correspond sûrement pas à $L$. (D.) halterella — et est inutilisable.

Fig. 12 à 15 effectuées sur la demande de M. H. Bangerter de Berne qui a fourni les spécimens.

Provenance : Clavadel sur Davos à $1900 \mathrm{~m}$ (Canton de Graubünden, Suisse) $(3 \hat{o}, 3$ q $)$.

\section{L. (D.) sericata Meigen 1830 .}

Les figures de DE MEIJERE [1919] corespondent sans doute à l'espèce étudiée ici (fig. 16 et 17) ainsi probablement que le schéma de Pienre ' [1924].

Provenance : les bords de la rivière la Louge vers $200 \mathrm{~m}$, dans les environs de Toulouse (Haute-Garonne), 7 et 21-IV-1968 (2 $\hat{o}), 25-I V-1969$ (1 $\hat{\delta})$. 

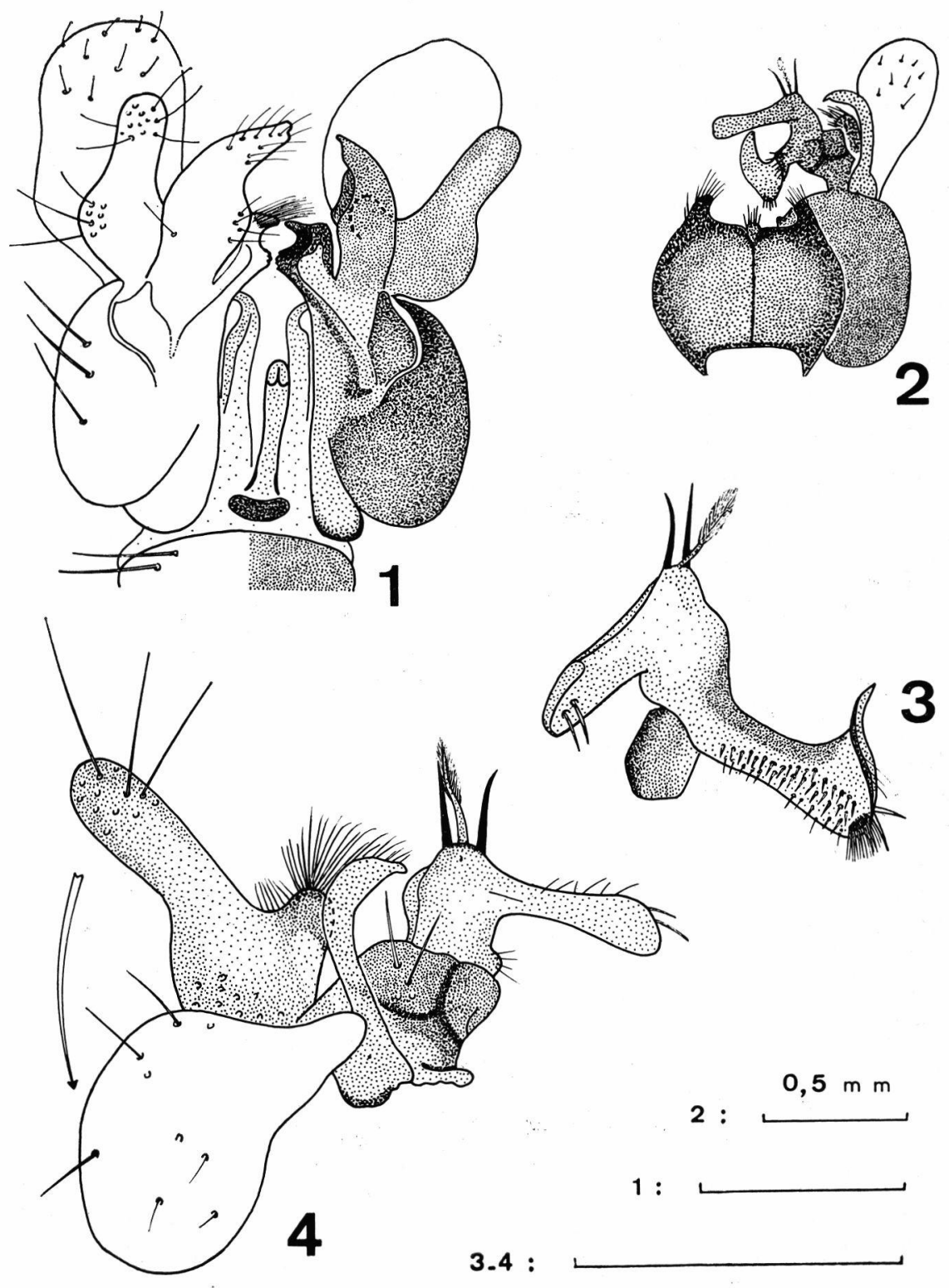

Fig. 1 à 4. - Genitalia ô de Limonia (Dicranomyia) stigmatica MeIGen. Fig. 1 : vue centrale; fig. 2 : vue dorsale; fig. 3 : appendice medio-dorsal droit, côté interne; fig. 4 : partie distale du demi-forceps droit, dernier article rabattu vers le bas. 

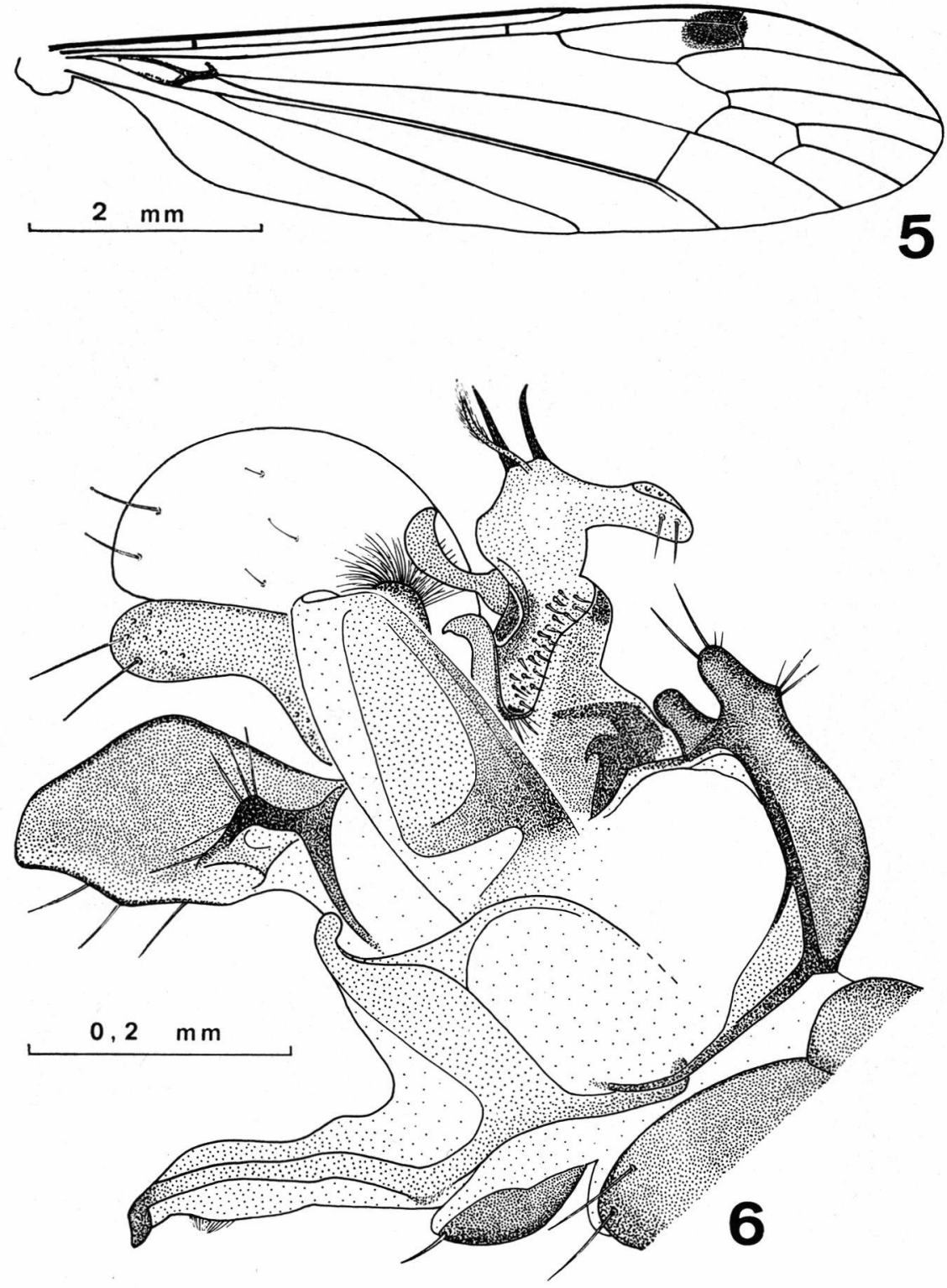

Fig. 5 et 6. - L. (D.) stigmatica Meigen. Fig. 5 : aile; fig. 6 : genitalia $\hat{o}$ en vue de profil, demi-forceps droit retiré. 


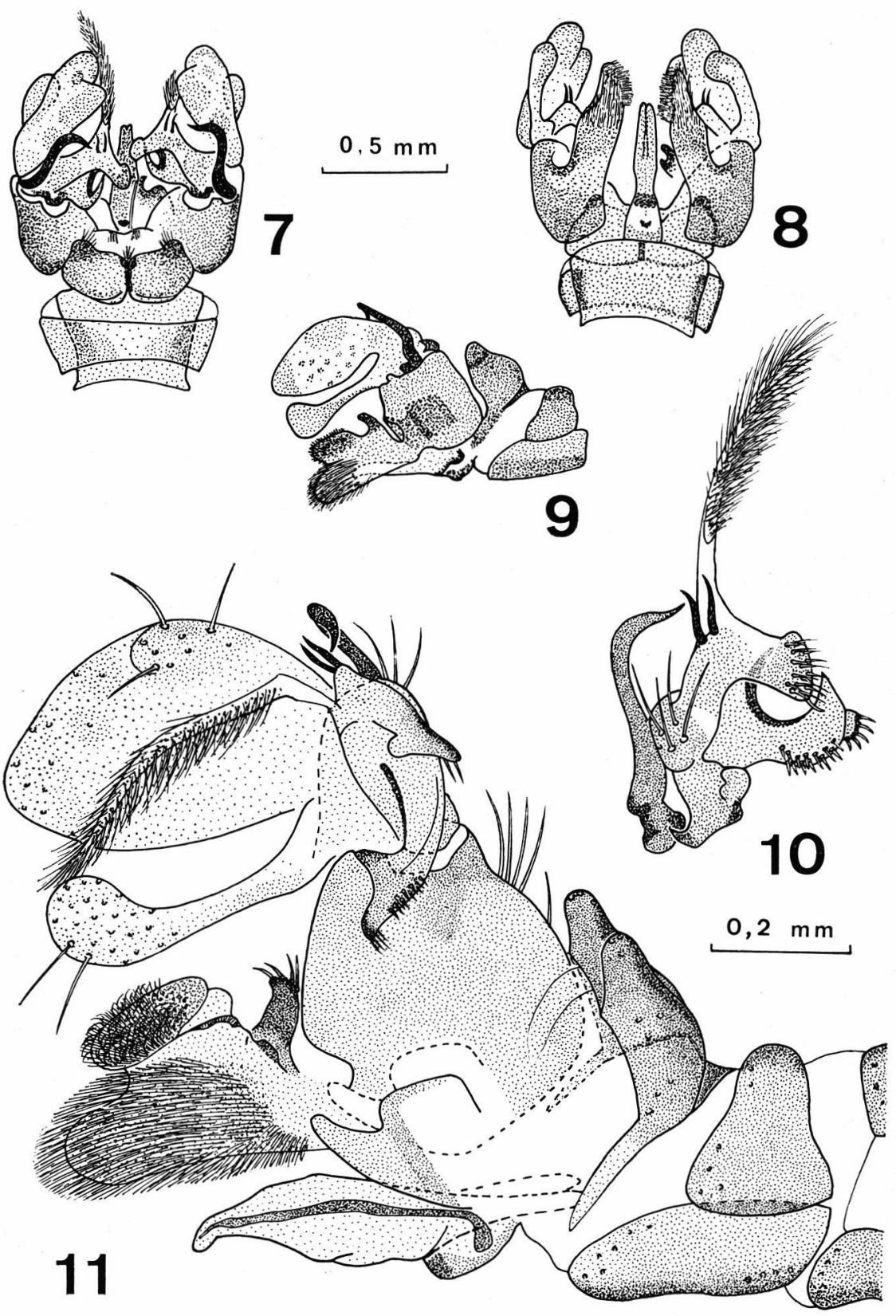

FIG. 7 à 11. - Genitalia $\hat{o}$ de L. (D.) lackschewitzi Edwards. Fig. 7 : vue dorsale; fig. 8 : vue ventrale; fig. 9 : vue de profil; fig. 10 : appendice médiodorsal droit et crochet; fig. 11 : semi-profil, demi-forceps droit retiré. 


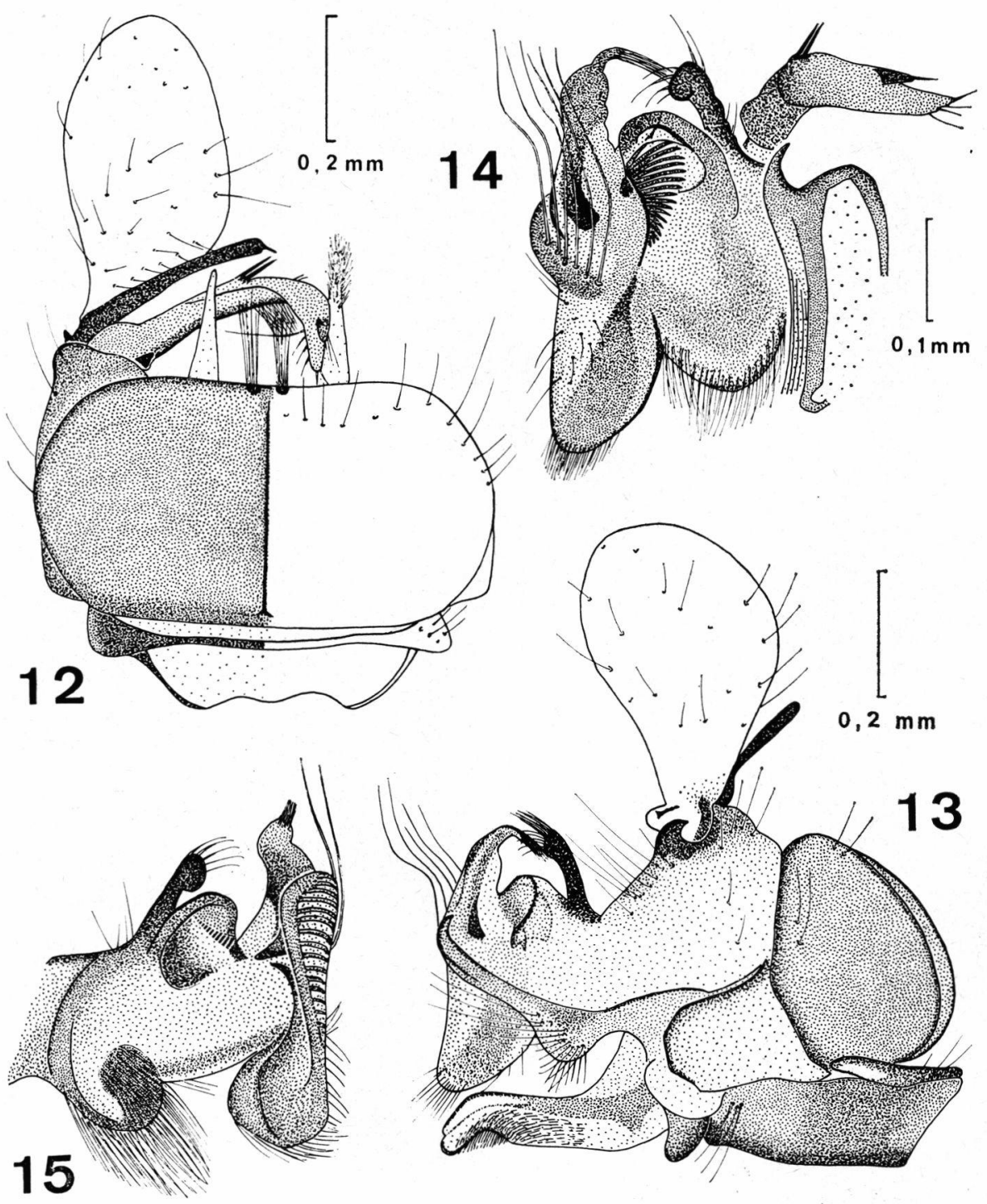

FIg. 12 à 15. - Genitalia ô de L. (D.) halteralla Edwards. Fig. 12 : vue dorsale; fig. 13 : vue de profil; fig. 14 : profil interne partiel d'un autre specimen, demi-forceps droit retiré, appendice terminal replié; fig. 15 : appendice terminal droit déroulé, vue interne. 


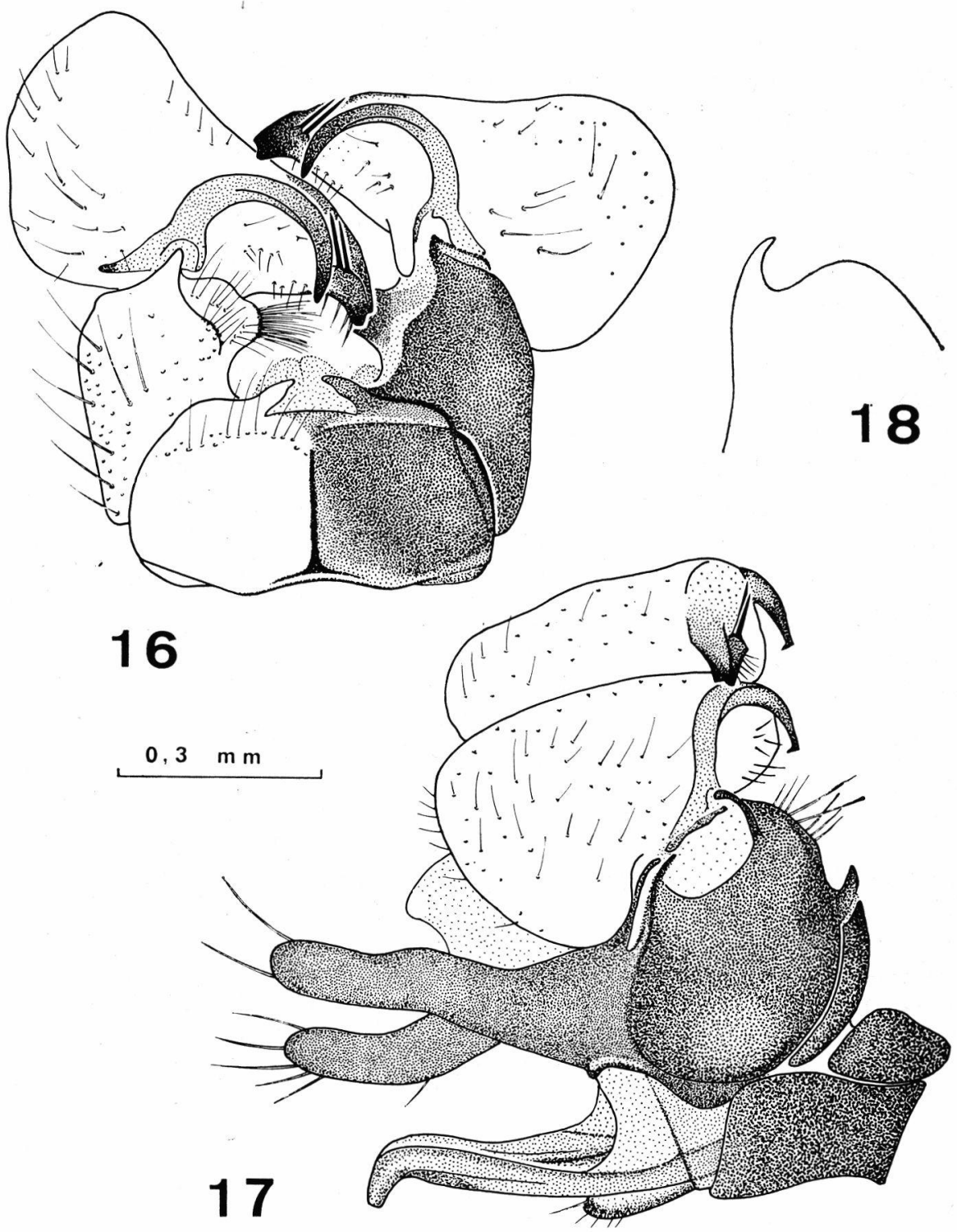

Fig. 16 à 18. - Genitalia ô de $L$. (D.) sericata Meigen. Fig. 16 : vue dorsale; fig. 17 : semi-profil droit; fig. 18 : paramère de profil. 


\section{RÉSUME}

Les genitalia $\hat{o}$ de quatre espèces du genre Limonia (Dicranomyia) peu connues et mal caractérisées sont figurés en détail.

\section{SUMMARY}

The genitals of four species of the genus Limonia (Dicranomyia) hitherto little known and insufficiently described are figured in detail.

\section{ZUSAMMENFASSUNG}

Die Genitalien von vier Arten der Gattung Limonia (Dicranomyia), bisher wenig bekannt und unzureichend beschrieben, werden eingehend dargestellt.

\section{TRAVAOX GITES}

Bangerter (H.). 1948. - Neue Diptera nematocera. Mitt. schweiz. ent. Ges., Bern, 21 (1) : 185-192.

CoE (R. L.). 1950. - Family Tipulidae. In Handbooks for the Identification of British Insects. IX, $2: 1-66$.

Edwards (F. W.). 1928. - The Nematocerous Diptera of Corsica. Encycl. ent., Paris, B III, Diptera 4 : 157-189.

Edwards (F.W.). 1938. - British Short-palped Crane-flies. Taxonomy of adults. Trans. Soc. Br. Ent., 5 : 1-168.

Lackschewitz (P.) \& Pagast (F.). 1940 à 1942. - 16. Limoniidae. In Lindner E., Die Fliegen der palaearktischen Region. 135: 1940, $139: 1941$ et $145: 1942$. Stuttgart.

Meijere (J. C. H. De). 1919 (1920). - Studien über palaearktische, vorwiegend holländische, Limnobiiden, insbesondere über ihre Kopulationsorgane. Tijdschr. Ent., 62 : 52-97 + IX pl.

Pierre (C.). 1924. - Diptères : Tipulidae. In Faune de France. 8. Paris. 